\title{
Serum soluble PD-1 plays a role in predicting infection complications in patients with acute pancreatitis
}

\section{Xingxing Yu | Yu Pan | Qinglin Fei | Xianchao Lin | Zhijiang Chen | Heguang Huang (i)}

Department of General Surgery, Fujian Medical University Union Hospital, Fuzhou, China

\section{Correspondence}

Heguang Huang, Department of General Surgery, Fujian Medical University Union Hospital, \#29 Xinquan Rd, 350001

Fuzhou, China.

Email: heguanghuang22@163.com

\section{Funding information}

Sailing of Fujian Medical University, Grant/Award Number: 2017XQ2024; Joint Funds of Scientific and Technological Innovation Program of Fujian Province, Grant/Award Number: 2017Y9059; Medical Center of Minimally Invasive Technology of Fujian Province, Grant/Award Numbers: 171, 2017 and 4, 2017

\begin{abstract}
Background: Most of acute pancreatitis (AP) are mild and self-limiting, however, $15 \%-20 \%$ of patients develop severe acute pancreatitis (SAP) or moderately acute pancreatitis (MSAP) with local or systemic complications. Infection complications (ICs) result in $40 \%-70 \%$ morbidity and high mortality rates among SAP and MSAP patients. It is more important to early recognize of ICs of MSAP or SAP. Several studies have indicated that serum soluble programmed cell death protein (SPD-1) or programmed cell death 1 ligand (sPD-L1) levels were higher in patients with severe sepsis than in healthy volunteers and have a predictive capacity for mortality. However, the role of serum SPD-1/sPD-L1 in AP remains unclear. This study aimed to investigate whether the ICs of AP patients is associated with their sPD-1 and sPD-L1 levels, which were determined via enzyme-linked immunosorbent assay of peripheral blood samples from $63 \mathrm{MSAP}$ and SAP patients and 30 healthy volunteers.
\end{abstract}

Results: The serum SPD-1 levels in AP patients on Days 1, 3, and 10 after onset were significantly increased in a time-dependent manner compared with that in healthy volunteers. Moreover, the AP patients with ICs had significantly higher serum sPD-1 levels than the AP without ICs. While serum SPD-L1 levels in AP were similar to that in healthy volunteers. Besides, serum levels of sPD-1/sPD-L1 were negatively correlated with circulating lymphocytes. Univariate and multivariate regression analyses showed that the upregulated serum sPD-1 level was an independent risk factor for ICs in AP. The area under the receiver operating characteristics curve indicated that

\footnotetext{
Abbreviations: AP, acute pancreatitis; APACHE II, Acute Physiology and Chronic Health Evaluation II; BISAP, Bedside Index for Severity in Acute Pancreatitis; HCT, hematocrit; IQR, interquartile range; LMR, lymphocyte-monocyte ratio; MSAP, moderately severe acute pancreatitis; NLR, neutrophil-lymphocyte ratio; PLR, platelet-lymphocyte ratio; SAP, severe acute pancreatitis; sPD-1, serum soluble programmed cell death; sPD-L1, serum soluble programmed cell death protein ligand 1; WBC, white blood cell.

Xingxing Yu and Yu Pan contributed equally to this study.

This is an open access article under the terms of the Creative Commons Attribution License, which permits use, distribution and reproduction in any medium, provided the original work is properly cited.

(c) 2020 The Authors. Immunity, Inflammation and Disease published by John Wiley \& Sons Ltd
} 
combination with Acute Physiology and Chronic Health Evaluation II score and serum SPD-1 level had a high accuracy in predicting ICs in AP.

Conclusion: Serum sPD-1/sPD-L1 may be involved in the immunosuppressive process in AP. Moreover, the serum sPD-1 level may be an independent risk factor for predicting ICs in AP patients.

\section{K E Y W O R D S}

acute pancreatitis, immunosuppression, infection complication, PD-1, PD-L1

\section{1 | BACKGROUND}

Acute pancreatitis (AP) is a common acute abdomen in general surgery, and most of the APs are mild and selflimiting, without complications and only needing a short hospitalization. ${ }^{1}$ However, $15 \%-20 \%$ of patients develop severe acute pancreatitis (SAP) or moderately severe acute pancreatitis (MSAP) with local or systemic complications, which has a high mortality. ${ }^{2,3}$ The main reason for the high mortality among SAP and MSAP patients is the infection complications (ICs), morbidity for which can be approximately $40 \%-70 \% .{ }^{4-6}$ It's required that early identification of SAP and MSAP patients at risk of developing ICs.

Studies showed that early immunosuppression of SAP has led to the occurrence of systemic ICs and multiple organ failure. ${ }^{7,8}$ Programmed cell death protein (PD-1) is a co-inhibitory molecule belonging to the CD28 family, mainly expressed in activated $\mathrm{T}$ lymphocytes, natural killer $\mathrm{T}$ cells, and bone marrow cells. ${ }^{9,10}$ The programmed cell death 1 ligand (PD-L1) is a ligand for PD-1 expressed on antigen-presenting and hematopoietic cells. ${ }^{9}$ The PD-1/PD-L1 pathway has been shown to regulate lymphocyte proliferation and apoptosis and play an important role in immune regulation. ${ }^{11-13}$ Previous studies had shown that PD-1 and PD-L1 exist in two forms: cell membrane-bound and soluble forms. ${ }^{14}$ Soluble PD-1 and PD-L1 (sPD-1/sPD-L1) can be detected in human serum. SPD-1 may promote T-cell responses by inhibiting the PD-1/PD-L1 signaling pathways, while excessive sPD-1 may lead to immunosuppression ${ }^{14}$; sPDL1 was released into the blood by the surface of PD-L1expressing cells that may reflect PD-L1 levels. ${ }^{15}$ Additionally, sPD-L1 may retain immunosuppression induction. ${ }^{15} \mathrm{~A}$ recent study revealed that $\mathrm{PD}-1$ expression in peripheral T cells and PD-L1 expression in monocytes increased significantly in sepsis patients than in healthy controls, ${ }^{16}$ and in AP patients with ICs than the patients without ICs. ${ }^{17}$ Several studies have indicated that serum sPD-1/sPD-L1 levels were higher in patients with severe sepsis than in healthy volunteers and had a predictive capacity for mortality. ${ }^{18,19}$ However, the relationship between serum sPD-1/sPD-L1 levels and ICs in AP has not been certified. Furthermore, serum sPD-1/sPD-L1 expression is easy to examine and has potential applications.

In this study, we investigated the levels of serum SPD1/sPD-L1 in SAP and MSAP patients and healthy volunteers to understand the association of these parameters with immune status and ICs in AP patients.

\section{2 | METHODS}

Peripheral blood was obtained from 63 patients with MSAP or SAP at Fujian Medical University Union Hospital, Fuzhou, China, from October 2017 to April 2019. Patient inclusion criteria included: (1) patients with MSAP or SAP, according to the 2012 edition of the Atlanta Convention AP classification criteria ${ }^{20}$; (2) aged 18 years or older; (3) admitted to the hospital within $48 \mathrm{~h}$ of onset. Exclusion criteria included: patients (1) with mild AP; (2) treated for $<10$ days; (3) with chronic pancreatitis, pregnancy, breastfeeding, acute and chronic hepatitis, endstage liver and kidney disease, immunodeficiency disease, and malignant tumor; (4) who had received immunosuppressive therapy. All patients were followed until discharge or hospital mortality. Patient baseline characteristics, Bedside Index for Severity in Acute Pancreatitis (BISAP), Ranson, and Acute Physiology and Chronic Health Evaluation II (APACHE II) scores were collected and recorded. Patient characteristics were collected and are shown in Table 1. This study was approved by the Committee for the Ethical Review of Research, Fujian Medical University Union Hospital.

Definition of ICs: infected pancreatic necrosis, bacteremia, pneumonia, infectious ascites, or urinary tract infections during admission. The diagnostic criteria for infected pancreatic necrosis were "positive for peripancreatic effusion or pancreatic necrosis tissue culture" obtained at the first pancreatic perivascular drainage or the first surgical treatment. The diagnostic criterion for 
TABLE 1 Characteristics of AP patients

\begin{tabular}{|c|c|}
\hline Characteristics & Data $(n=63)$ \\
\hline Age (years, mean $\pm S D$ ) & $51.08 \pm 13.56$ \\
\hline Sex ( $n$, female/male) & $29 / 34$ \\
\hline \multicolumn{2}{|l|}{ Severity of AP, $n(\%)$} \\
\hline Moderately severe & $35(55.6)$ \\
\hline Severe & $28(44.4)$ \\
\hline \multicolumn{2}{|l|}{ Etiology of AP, $n(\%)$} \\
\hline Biliary & $19(30.2)$ \\
\hline Hypertriglyceridemia & $24(38.1)$ \\
\hline Alcoholicity & $4(6.3)$ \\
\hline Other & $16(25.4)$ \\
\hline Ranson score, median (IQR) & $2.0(1-3)$ \\
\hline BISAP score, median (IQR) & $2.0(1-2)$ \\
\hline APACHE II score, median (IQR) & $10.0(8-15)$ \\
\hline \multicolumn{2}{|l|}{ Infection complications, $n$} \\
\hline Pneumonia & 36 \\
\hline Infected necrosis & 20 \\
\hline Bacteremia & 3 \\
\hline \multicolumn{2}{|l|}{ Organ dysfunction, $n$} \\
\hline Respiratory & 29 \\
\hline Cardiovascular & 10 \\
\hline Renal & 11 \\
\hline \multicolumn{2}{|l|}{ Interventions, $n$} \\
\hline Surgical & 29 \\
\hline Mechanical ventilation & 7 \\
\hline Renal replacement therapy & 3 \\
\hline Hospital mortality, $n(\%)$ & $2(3.2)$ \\
\hline
\end{tabular}

Abbreviations: AP, acute pancreatitis; APACHE II, Acute Physiology and Chronic Health Evaluation II; BISAP, The Bedside Index for Severity in Acute Pancreatitis; IQR, interquartile range.

bacteremia was "positive for blood culture." Diagnostic criteria for pneumonia included: (1) newly developed cough, or symptoms of the original respiratory disease, with purulent sputum, with or without chest pain; (2) fever $\geq 38^{\circ} \mathrm{C}$; (3) lung consolidation signs and/or wet rales; (4) white blood cell (WBC) $>10 \times 10^{9} / \mathrm{L}$ or $<4 \times 10^{9} / \mathrm{L}$ with or without nuclear left shift; (5) lung imaging suggests patchy infiltrating shadow or interstitial changes with or without pleural effusion. Any of the above (1) to (4) plus the fifth item can lead to a diagnosis, except for tuberculosis, lung cancer, noninfectious pulmonary interstitial disease, pulmonary edema, atelectasis, pulmonary embolism, pulmonary eosinophilic infiltration, and pulmonary vasculitis. The diagnostic criterion for infectious ascites is the positive ascites specimen obtained during the first abdominal puncture drainage or the first surgery. Diagnostic criteria (and confirmation) for urinary tract infections included: bacterial colony count $\geq 10^{5} / \mathrm{ml}$ and WBC count $>10 / \mathrm{HP}$ following centrifugation of urine collected midstream. Multiple infections in the same patient were considered one endpoint. ${ }^{17}$

\section{$2.1 \quad$ Blood samples}

Peripheral blood samples were obtained from 30 healthy volunteers (control) and AP patients on Days 1, 3, and 10 after admission. Serum samples were collected immediately after centrifugation at $3000 \mathrm{rpm}$ for $15 \mathrm{~min}$ at $4^{\circ} \mathrm{C}$, and stored at $-80^{\circ} \mathrm{C}$ for subsequent analysis.

\section{2 | Serum SPD-1 and SPD-L1 analysis}

Serum sPD-1/sPD-L1 was quantified using the human sPD-1/sPD-L1 enzyme-linked immunosorbent assay (ELISA) kit $\left(\right.$ RayBio $\left.^{\circledR}\right)$. Serum sPD-1/sPD-L1 levels were measured in duplicates and analyzed according to manufacturers' recommendations. A 1:50 dilution was used for all the samples. The nonlinear standard curve was constructed based on polynomial regression $($ degree $=2$ ).

\section{3 $\quad$ Statistical analysis}

SPSS 22.0 software (SPSS Inc.) was used for statistical analysis. Results are presented as medians and interquartile ranges (IQRs) or mean $\pm S D$, and categorical variables are shown as frequency and percentage. The normal distribution of all variables was tested using the Shapiro-Wilk test. $\chi^{2}$ or Fisher's tests was used for twocategory variables. The independent sample $t$ test was used to compare variables that conform to the normal distribution, and the Mann-Whitney $U$ test to compare variables that are not normally distributed. The correlation was assessed by a Spearman rank test. The concentrations at different times (Days 1, 3, and 10) in each group were compared using one-way repeated measures analysis of variance. A two-category univariate logistic regression analysis was performed to assess the correlation between the variables (Table 2) and AP infectious complications. Then only the significant differences in univariate analysis were using multivariate stepwise regression analysis of variables. The area under the receiver operating characteristics (ROCs) curve (AUC) was used to estimate the accuracy of the predicted model, and the AUC was bilaterally $p<.05$. Figures were prepared using GraphPad Prism version 6.0 (GraphPad Software). 
TABLE 2 Univariate and multivariate regression analysis of variables for ICs of AP

\begin{tabular}{|c|c|c|c|c|}
\hline \multirow[b]{2}{*}{ Variable } & \multicolumn{2}{|l|}{ Univariate analysis } & \multicolumn{2}{|c|}{ Multivariate analysis } \\
\hline & $\overline{\text { OR (95\% CI) }}$ & $p$ value & $\overline{\text { OR (95\% CI) }}$ & $p$ value \\
\hline Age & $1.016(0.979-1.055)$ & .395 & & \\
\hline $\mathrm{HCT}(\%)$ & $0.908(0.842-0.980)$ & .013 & $0.921(0.842-1.007)$ & .071 \\
\hline APACHE II score & $1.420(1.134-1.776)$ & .002 & $1.281(1.008-1.629)$ & .043 \\
\hline Neutrophil count on Day 1 & $0.987(0.898-1.084)$ & .783 & & \\
\hline Serum sPD- 1 levels on Day 1 & $1.002(0.997-1.007)$ & .428 & & \\
\hline Serum sPD-1 levels on Day 3 & $1.013(1.005-1.021)$ & .002 & $1.009(1.001-1.018)$ & .029 \\
\hline
\end{tabular}

Note: Bold values represent factors greater than .05 in the $p$ value.

Abbreviations: AP, acute pancreatitis; APACHE II, Acute Physiology and Chronic Health Evaluation II; CI, confidence interval; HCT, hematocrit; IC, infection complication; OR, odds ratio.

\section{3 | RESULTS}

\subsection{Characteristics of the patients}

According to the revised Atlanta classification, ${ }^{20}$ a total of 63 patients with AP (28 SAP and 35 MSAP patients) were included in this study, with an average age of $51.08 \pm 13.56$ years. For the classification of AP etiology, hypertriglyceridemia-induced pancreatitis was the main cause, accounting for $38.1 \%$, followed by biliary $30.2 \%$, alcoholicity $6.3 \%$, and other factors $25.4 \%$. All AP patients underwent three AP-related scoring system after admission, including the BISAP (assessment after 24-h admission), Ranson (48-h), and APACHE II (48-h) scores. The clinical characteristics of these patients are shown in Table 1.
(A)

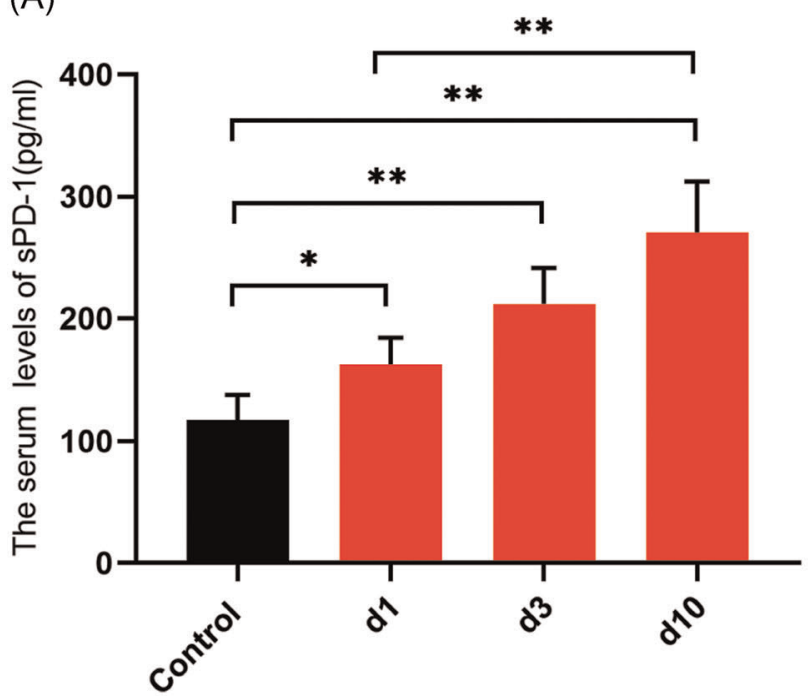

(B)

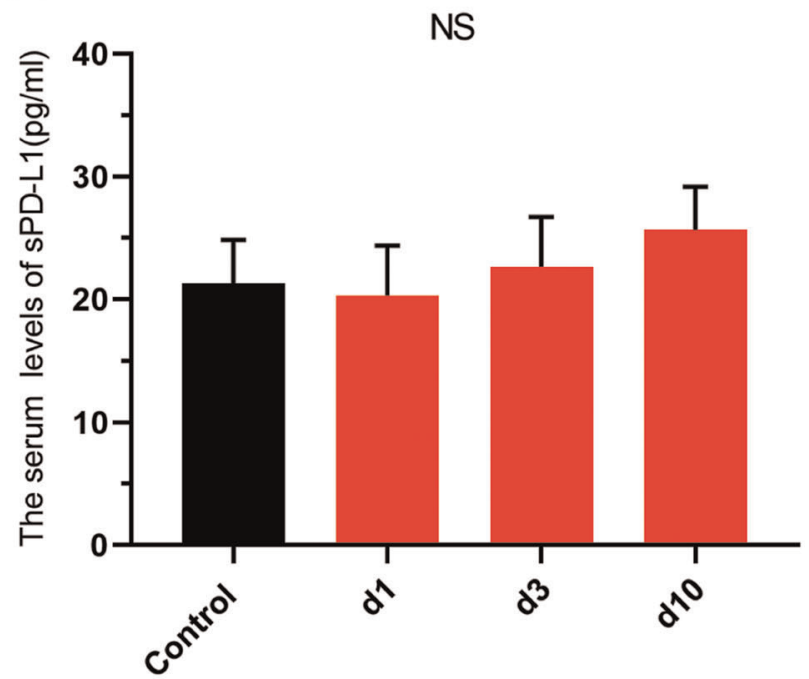

FI G U RE 1 The serum sPD-1 and sPD-L1 levels in patients with acute pancreatitis (AP). (A) sPD-1 and (B) sPD-L1 were measured in peripheral blood from healthy volunteers (control, $n=30)$ and patients with AP $(n=63)$ on Day 1 (d1), Day 3 (d3), and Day 10 (d10) after onset. ${ }^{*} p<.05, * * p<.01$ 


\section{2 | Serum SPD-1 and SPD-L1 levels in patients of $A P$}

Serum levels of SPD-1 and sPD-L1 were measured in patients of AP on Day 1 (d1), Day 3 (d3), and Day 10 (d10) after admission. The serum sPD-1 levels in AP patients on $\mathrm{d} 1, \mathrm{~d} 3$, and $\mathrm{d} 10$ were significantly elevated compared with that in healthy controls $(p<.05, p<.01$, $p<.01$; Figure 1A). Moreover, serum sPD-1 level in AP patients was upregulated in a time-dependent manner, and was most elevated on Day 10 compared with that on Day $1(p<.01$; Figure $1 \mathrm{~A})$. However, serum sPD-L1 levels on d1, d3, and d10 in AP patients were similar to that in healthy controls (Figure 1B).

\section{3 | Correlation between clinical indicators and serum SPD-1/sPD-L1 levels}

We further investigated the relationship between clinical indicators and serum SPD-1/sPD-L1 levels. We observed that the serum levels of sPD-1 and SPD-L1 on Day 10 were both negatively correlated with lymphocyte count $(r=-.335, p=.015 ; r=-.294, p=.035$; Table 3$)$, whereas the serum level of SPD-1 on Day 1 was positively correlated with lymphocyte-monocyte ratio (LMR; $r=.269, p=.034$; Table 3 ). Moreover, the serum level of sPD-1 on Days 3 and 10 was negatively associated with the hematocrit (HCT; $r=-.289, p=.021$; $r=-.331, p=.016$ ).

\subsection{Correlation between serum sPD-1/ SPD-L1 levels and ICs of AP}

To investigate the relationship between ICs of AP and serum SPD-1/sPD-L1 levels, all patients were divided into two groups: AP with $(n=36)$ and without $(n=27)$ ICs. We found that APACHE II scores were significantly higher in the AP with ICs group than in the AP without ICs group $(p<.001$; Table 4$)$. Whereas the HCT was significantly higher in the AP without ICs group than in the AP with ICs group ( $p=.003$; Table 4$)$. The AP with ICs group had significantly higher serum SPD-1 levels on Days 3 and 10 than the AP without ICs group $(p<.001$, $p<.001$; Table 4). However, there were no significant differences between AP with ICs group and AP without ICs group with regard to serum SPD-L1 levels.

TABLE 3 Correlation between traditional clinical indicators and serum sPD-1/sPD-L1

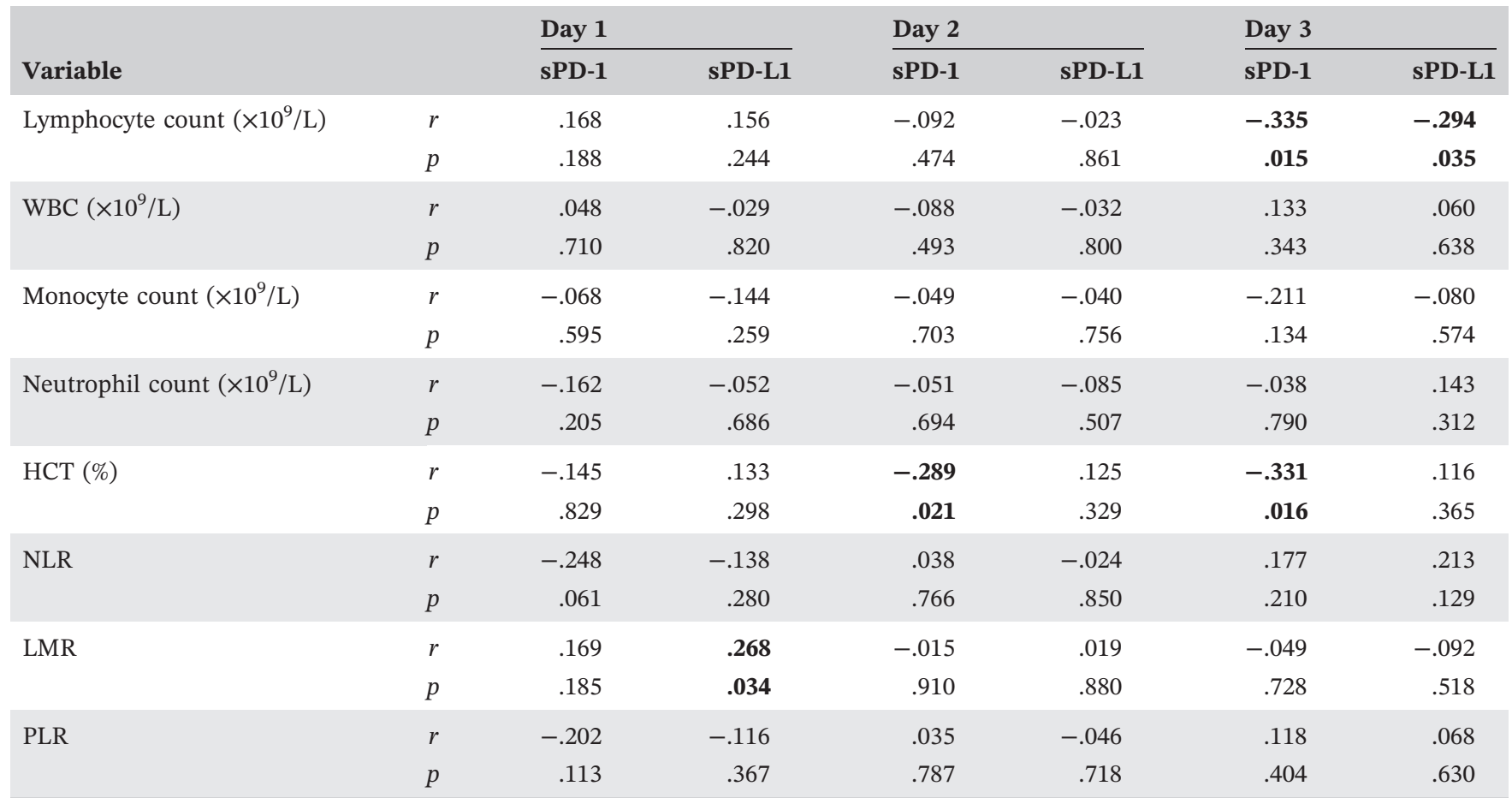

Note: Bold values represent factors greater than .05 in the $p$ value.

Abbreviations: HCT, hematocrit; LMR, lymphocyte-monocyte ratio; NLR, neutrophil-lymphocyte ratio; PLR, platelet-lymphocyte ratio; WBC, white blood cell. 
TABLE 4 Clinical indicators of patients with AP with or without ICs

\begin{tabular}{|c|c|c|c|}
\hline & AP with IC $(n=36)$ & AP without IC $(n=27)$ & $p$ value \\
\hline Age (years) & $52.33 \pm 13.76$ & $49.41 \pm 13.37$ & .436 \\
\hline Male/female $(n)$ & $24 / 12$ & $10 / 17$ & - \\
\hline WBC count $\left(\times 10^{9} / \mathrm{L}\right)$ & $12.42 \pm 5.41$ & $12.97 \pm 5.67$ & .755 \\
\hline Neutrophil count on Day $1\left(\times 10^{9} / \mathrm{L}\right)$ & $9.98 \pm 5.23$ & $10.35 \pm 5.54$ & .824 \\
\hline Lymphocyte count on Day $1\left(\times 10^{9} / \mathrm{L}\right)$ & $1.20 \pm 0.58$ & $1.26 \pm 0.66$ & .760 \\
\hline $\operatorname{PLT}\left(\times 10^{9} / \mathrm{L}\right)$ & $282.06 \pm 116.47$ & $239.44 \pm 105.32$ & .090 \\
\hline $\mathrm{HCT}(\%)$ & $31.66 \pm 7.79$ & $36.79 \pm 6.75$ & .003 \\
\hline sPD-L1 levels on Day 1 (pg/ml) & $25.83 \pm 16.01$ & $30.78 \pm 21.51$ & .413 \\
\hline sPD-L1 levels on Day $3(\mathrm{pg} / \mathrm{ml})$ & $31.02 \pm 17.61$ & $29.49 \pm 16.63$ & .890 \\
\hline sPD-L1 levels on Day $10(\mathrm{pg} / \mathrm{ml})$ & $27.58 \pm 15.02$ & $33.75 \pm 14.81$ & .060 \\
\hline
\end{tabular}

Note: Bold values represent factors greater than .05 in the $p$ value.

Abbreviations: AP, acute pancreatitis; APACHE II, Acute Physiology and Chronic Health Evaluation II; HCT, hematocrit; IC, infection complication; PLT, platelet; WBC, white blood cell.

\section{5 | Serum sPD-1 may be an independent factor for predicting ICs in AP}

To determine the predictive effect of age, APACHE II scores, HCT, and lymphocyte, monocyte, and neutrophil counts on Day 1, and serum SPD-1 and SPD-L1 levels on Days 1 and 3 for ICs, we performed a logistic regression analysis. Univariate analysis demonstrated that HCT (odds ratio [OR], 0.908; 95\% CI, 0.842-0.980; $p=.013$ ), APACHE II score (OR, 1.420; 95\% CI, 1.134-1.776; $p=.002$ ), and serum sPD-1 level on Day 3 (OR, 1.013; 95\% CI, $1.005-1.021 ; p=.002)$ were significantly associated with ICs of AP (Table 2). Furthermore, we performed multivariate analysis to evaluate $\mathrm{HCT}$, APACHE II score, and serum sPD-1 level on Day 3 as independent predictors of ICs. The results suggested that serum SPD-1 levels on Day 3 (OR, 1.009; 95\% CI, 1.001-1.018; $p=.029$ ) and the APACHE II score (OR, 1.281; 95\% CI, $1.008-1.629 ; p=.043)$ were independent risk predictors of ICs in AP (Table 2).

To evaluate the predictive accuracy of serum SPD-1 levels on Day 3 and the APACHE II score for ICs in AP patients, the ROCs curve analysis was performed. The AUC values for serum sPD-1 levels on Day 3 and APACHE II score were 0.796 (95\% CI, 0.681-0.911; $p<.001$ ) and 0.769 (95\% CI, 0.649-0.889; $p<.001$; Table 5 and Figure 2). By combining these two variables, a high accuracy for AP IC prediction was achieved $(\mathrm{AUC}=0.826 ; 95 \% \mathrm{CI}, 0.721-0.931 ; p<.001$; Table 5 and Figure 2).

\section{4 | DISCUSSION}

MSAP and SAP could develop into immunosuppression, leading to secondary infection and pancreatic necrosis. $^{21,22}$ Our study showed that compared with healthy volunteers, serum SPD-1 levels in the MSAP and

TABLE 5 AUCs of various parameters for predicting ICs in AP patients

\begin{tabular}{llll} 
Variable & AUC & $\boldsymbol{p}$ value & $\mathbf{9 5 \%} \mathbf{~ C l}$ \\
\hline APACHE II score & 0.769 & $<.001$ & $0.649-0.889$ \\
Serum sPD-1 levels on Day 3 & 0.796 & $<.001$ & $0.681-0.911$ \\
$\begin{array}{l}\text { Combination of above two } \\
\quad \text { various }\end{array}$ & 0.826 & $<.001$ & $0.721-0.931$ \\
\hline
\end{tabular}

Abbreviations: AP, acute pancreatitis; APACHE II, Acute Physiology and Chronic Health Evaluation II; AUC, area under the curve; CI, confidence interval; IC, infection complication. 
-.-. Day 3 serum SPD-1 levels AUC : 0.796

APACHE II score AUC : 0.769

- Combined AUC: 0.826

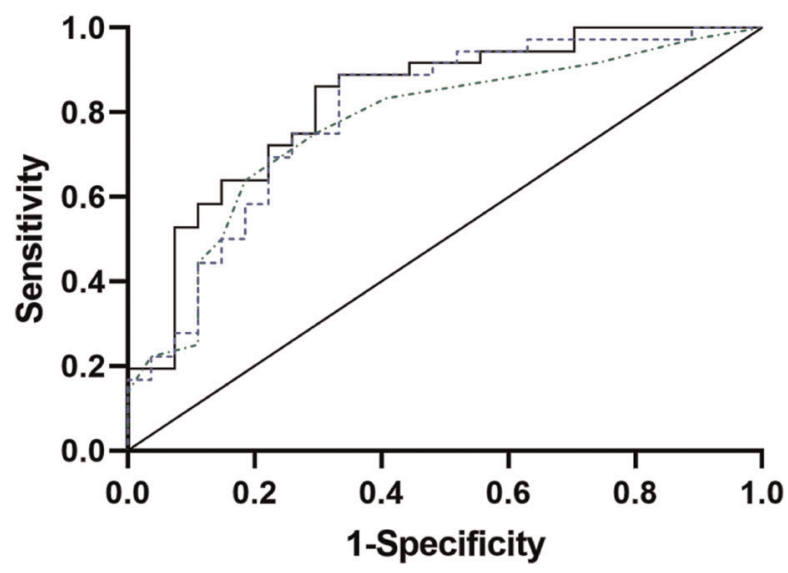

FIG URE 2 The area under the ROC curve (AUC) was used to estimate the accuracy of the predicted model. AUC of serum SPD-1 level on Day 3: 0.796; AUC of Acute Physiology and Chronic Health Evaluation II score: 0.769; AUC of combined: 0.826

SAP patients increased continuously during the early course of the disease, especially the patients with ICs. Moreover, elevated SPD-1 level was associated with enhanced occurrence of ICs. Studies showed that serum SPD-1 may promote T-cell responses by inhibiting the PD-1/PD-L1 signaling pathway, but the continuously excessive level of serum sPD-1 may serve as an antibody to block the PD-1/PD-L1 pathway, which leads to the aberrant activation and proliferation of $\mathrm{T}$ cells. ${ }^{14,23}$ The uncontrolled immune regulation resulted in hyperimmune behavior in the early stage of SAP, however, with the consumption of lymphocytes, the hyperimmune status transformed into immunosuppression and increased the incidence of ICs. Finally, a marked increase in SPD-1 levels may represent more severe immune damage in patients. ${ }^{18}$ In addition, sPD-L1 may retain the immunosuppressive condition and continuously increased SPD-L1 ultimately aggravates immunosuppression. ${ }^{15,24}$ Hence, serum sPD-1/sPD-L1 levels may play an important role in monitoring the immune status of AP patients and predicting ICs and prognosis.

Furthermore, our data indicated that serum sPD-1/ sPD-L1 levels of AP patients are associated with LMR, HCT, and lymphocyte counts. Immune dysfunction in AP patients may be caused by decreased peripheral blood lymphocytes. ${ }^{24}$ The decreased expression of human leukocyte antigen-DR (HLA-DR) on monocytes may lead to early immunosuppression of AP. ${ }^{25}$ Moreover, serum SPD-L1 was reported to be involved in lymphocyte apoptosis. ${ }^{15}$ Our investigation of the correlation between serum SPD-1/sPD-L1 and clinical indicators revealed that dynamic monitoring of serum SPD-1/sPD-L1 levels in AP patients may reflect systemic immunologic functions in AP patients.

Previous studies indicated that BISAP, Ranson, and APACHE II scores could predict the mortality of AP patients with high accuracy ${ }^{26-28}$ and high Ranson, BISAP, and APACHE II scores were also associated with organ failure and complications in AP patients. ${ }^{29,30}$ In this study, we showed that the elevated serum SPD-1 level was an independent risk factor for ICs in patients of AP. Combination of APACHE II score and serum sPD-1 level may better predict ICs of AP patients. Depending on the prediction of SPD-1 in AP patients, the level of sPD-1 in different grades may use to guide the prevention and treatment of ICs in AP patients. The previous study pointed out that the combination of antiinflammatory and immune-stimulating therapies may be a new promising approach to AP immunotherapy. ${ }^{31}$ In the complex inflammatory response of $\mathrm{AP}$, a large number of pro-inflammatory cytokines such as TNF- $\alpha$ and IL- 6 were produced and maintained the proinflammatory-anti-inflammatory balance process of AP. In addition, pro-inflammatory cytokines, such as TNF $\alpha$ and IL-6, could modulate the level of SPD-1 in vitro and TNF-inhibitor therapy could modulate sPD-1 levels in the serum and synovial fluid of patients with inflammatory arthritis. ${ }^{32}$ Further study to reduce the production of SPD-1 through anti-pro-inflammatory cytokines may enhance the immune status and reduce the occurrence of ICs. However, there remain some limitations in this study, we investigated these variates in AP patients from a single center and the number of cases in this study was small. Further study involving a large cohort from multiple centers is needed to confirm these results.

\section{5 | CONCLUSIONS}

Serum sPD-1/sPD-L1 levels may be involved in the immunosuppressive process of AP, and SPD-1, which increases continuously in the peripheral blood of AP patients, maybe an independent risk factor for predicting ICs in AP patients, which is potentially applicable in determining or improving AP patient prognosis.

\section{ACKNOWLEDGMENTS}

The authors thank Yuanyuan Zhou for her kindly help. This study was supported by the Fund of Sailing of Fujian Medical University (2017XQ2024); Medical Center of Minimally Invasive Technology of Fujian Province (No. 171, 2017 and No. 4, 2017); Joint Funds of Scientific 
and Technological Innovation Program of Fujian Province (2017Y9059).

\section{CONFLICT OF INTERESTS}

The authors declare that there are no conflict of interests.

\section{ETHICS STATEMENT}

All procedures performed in studies involving human participants were in accordance with the Helsinki declaration. All patients whose blood samples were used in this study provided written informed consent, and the study was approved by the Committee for the Ethical Review of Research, Fujian Medical University Union Hospital.

\section{AUTHOR CONTRIBUTIONS}

Yu Pan, Xianchao Lin, and Heguang Huang conceived the concept. Heguang Huang supervised the study. Yu Pan, Qinglin Fei, Xingxing Yu designed and performed the experiments. Yu Pan, Xianchao Lin wrote the manuscript. All authors approved the manuscript.

\section{DATA AVAILABILITY STATEMENT}

The data that support the findings of this study are available from the corresponding author upon reasonable request.

\section{ORCID}

\section{Heguang Huang (D) http://orcid.org/0000-0003-1459-5546}

\section{REFERENCES}

1. Minkov GA, Halacheva KS, Yovtchev YP, Gulubova MV. Pathophysiological mechanisms of acute pancreatitis define inflammatory markers of clinical prognosis. Pancreas. 2015;44: 713-717.

2. Beger HG, Rau B, Isenmann R. Natural history of necrotizing pancreatitis. Pancreatology. 2003;3:93-101.

3. Tsui N, Zhao E, Li Z, et al. Microbiological findings in secondary infection of severe acute pancreatitis: a retrospective clinical study. Pancreas. 2009;38:499-502.

4. Cacopardo B, Pinzone M, Berretta S, et al. Localized and systemic bacterial infections in necrotizing pancreatitis submitted to surgical necrosectomy or percutaneous drainage of necrotic secretions. BMC Surg. 2013;13(suppl 2):S50. https:// doi.org/10.1186/1471-2482-13-S2-S50

5. Schmid SW, Uhl W, Friess H, Malfertheiner P, Buchler MW. The role of infection in acute pancreatitis. Gut. 1999;13(2): 311-316.

6. Renner IG, Savage WT, 3rd, Pantoja JL, Renner VJ. Death due to acute pancreatitis. A retrospective analysis of 405 autopsy cases. Dig Dis Sci. 1985;30(10):1005-1018.

7. Ueda $\mathrm{T}$, Takeyama $\mathrm{Y}$, Yasuda $\mathrm{T}$, et al. Immunosuppression in patients with severe acute pancreatitis. J Gastroenterol. 2006; 41(8):779-784.
8. Beger HG, Rau BM. Severe acute pancreatitis: clinical course and management. World J Gastroenterol. 2007;13(38):5043-5051. https://doi.org/10.3748/wjg.v13.i38.5043

9. Keir ME, Butte MJ, Freeman GJ, Sharpe AH. PD-1 and its ligands in tolerance and immunity. Annu Rev Immunol. 2008;26:677-704. https://doi.org/10.1146/annurev.immunol. 26.021607.090331

10. Gu YL. Immunosuppression and the infection in patients with early SAP. Front Biosci (Landmark Ed). 2013;18:892-900. https://doi.org/10.2741/4150

11. Chang K, Svabek C, Vazquez-Guillamet C, et al. Targeting the programmed cell death 1: programmed cell death ligand 1 pathway reverses $\mathrm{T}$ cell exhaustion in patients with sepsis. Crit Care. 2014;18(1):R3. https://doi.org/10.1186/cc13176

12. Zhang Y, Zhou Y, Lou J, et al. PD-L1 blockade improves survival in experimental sepsis by inhibiting lymphocyte apoptosis and reversing monocyte dysfunction. Crit Care. 2010;14(6):R220. https://doi.org/10.1186/cc9354

13. Latchman Y, Wood CR, Chernova T, et al. PD-L2 is a second ligand for PD-1 and inhibits $\mathrm{T}$ cell activation. Nat Immunol. 2001;2(3):261-268. https://doi.org/10.1038/85330

14. Dai S, Jia R, Zhang X, Fang Q, Huang L. The PD-1/PD-Ls pathway and autoimmune diseases. Cell Immunol. 2014; 290(1):72-79.

15. Frigola $\mathrm{X}$, Inman $\mathrm{BA}$, Lohse $\mathrm{CM}$, et al. Identification of a soluble form of B7-H1 that retains immunosuppressive activity and is associated with aggressive renal cell carcinoma. Clin Cancer Res. 2011;17:1915-1923.

16. Zhang Y, Li J, Lou J, et al. Upregulation of programmed death1 on $T$ cells and programmed death ligand- 1 on monocytes in septic shock patients. Crit Care. 2011;15:R70. https://doi.org/ 10.1186/cc10059

17. Pan $\mathrm{T}$, Zhou $\mathrm{T}$, Li L, et al. Monocyte programmed death ligand-1 expression is an early marker for predicting infectious complications in acute pancreatitis. Crit Care. 2017;21:186. https://doi.org/10.1186/s13054-017-1781-3

18. Zhao Y, Jia Y, Li C, Shao R, Fang Y. Predictive value of soluble programmed death-1 for severe sepsis and septic shock during the first week in an intensive care unit. Shock. 2019;51(3): 289-297. https://doi.org/10.1097/SHK.0000000000001171

19. Zhao Y, Jia Y, Li C, Fang Y, Shao R. The risk stratification and prognostic evaluation of soluble programmed death-1 on patients with sepsis in emergency department. Am J Emerg Med. 2018;36(1):43-48. https://doi.org/10.1016/j. ajem.2017.07.002

20. Banks PA, Bollen TL, Dervenis C, et al. Classification of acute pancreatitis-2012: revision of the Atlanta classification and definitions by international consensus. Gut. 2013;62(1): 102-111.

21. Naskalski JW1, Kusnierz-Cabala B, Kedra B, Dumnicka P, Panek J, Maziarz B. Correlation of peripheral blood monocyte and neutrophil direct counts with plasma inflammatory cytokines and TNF-alpha soluble receptors in the initial phase of acute pancreatitis. Adv Med Sci. 2007;52:129-134.

22. Pan Yu, Fang Haizong, Lu Fengchun, et al. Ulinastatin ameliorates tissue damage of severe acute pancreatitis through modulating regulatory T cells. J Inflamm (Lond). 2017;14:7. https://doi.org/10.1186/s12950-017-0154-7 
23. Greisen SR, Rasmussen TK, Stengaard-Pedersen K, et al Increased soluble programmed death-1 (sPD-1) is associated with disease activity and radiographic progression in early rheumatoid arthritis. Scand J Rheumatol. 2014;43(2): 101-108.

24. Takeyama Y, Takas K, Ueda T, Hori Y, Goshima M, Kuroda Y. Peripheral lymphocyte reduction in severe acute pancreatitis is caused by apoptotic cell death. J Gastrointest Surg. 2000;4(4): 379-387.

25. Mentula P, Kylänpää-Bäck ML, Kemppainen E, et al. Decreased HLA (human leucocyte antigen)-DR expression on peripheral blood monocytes predicts the development of organ failure in patients with acute pancreatitis. Clin Sci (Lond). 2003;105(4):409-417.

26. Hagjer S, Kumar N. Evaluation of the BISAP scoring system in prognostication of acute pancreatitis - a prospective observational study. Int J Surg. 2018;54(Pt A):76-81. https://doi.org/ 10.1016/j.ijsu.2018.04.026

27. Vasudevan S, Goswami P, Sonika U, Thakur B, Sreenivas V, Saraya A. Comparison of various scoring systems and biochemical markers in predicting the outcome in acute pancreatitis. Pancreas. 2018;47(1):65-71. https://doi.org/10. 1097/MPA.0000000000000957

28. Mounzer R, Langmead CJ, Wu BU, et al. Comparison of existing clinical scoring systems to predict persistent organ failure in patients with acute pancreatitis. Gastroenterology. 2012;142(7):1476-1482. https://doi.org/10.1053/j.gastro.2012. 03.005
29. Papachristou GI, Muddana V, Yadav D, et al. Comparison of BISAP, Ranson's, APACHE-II, and CTSI scores in predicting organ failure, complications, and mortality in acute pancreatitis. Am J Gastroenterol. 2010;105(2):435-441. https://doi.org/ 10.1038/ajg.2009.622

30. Kumar AH, Griwan MS. A comparison of APACHE II, BISAP, Ranson's score and modified CTSI in predicting the severity of acute pancreatitis based on the 2012 revised Atlanta Classification. Gastroenterol Rep (Oxf). 2018;6(2):127-131. https://doi. org/10.1093/gastro/gox029

31. Li J, Yang W-J, Huang L-M, Tang C-W. Immunomodulatory therapies for acute pancreatitis. World J Gastroenterol. 2014; 20(45):16935-16947. https://doi.org/10.3748/wjg.v20.i45.16935

32. Bommarito D, Hall C, Taams LS, Corrigall VM. Inflammatory cytokines compromise programmed cell death-1 (PD-1)mediated $\mathrm{T}$ cell suppression in inflammatory arthritis through up-regulation of soluble PD-1. Clin Exp Immunol. 2017;188(3): 455-466. https://doi.org/10.1111/cei.12949

How to cite this article: Yu X, Pan Y, Fei Q, Lin X, Chen Z, Huang H. Serum soluble PD-1 plays a role in predicting infection complications in patients with acute pancreatitis. Immun Inflamm Dis. 2021;9: 310-318. https://doi.org/10.1002/iid3.394 